\title{
NONSTABLE $K$-THEORY RESULTS FOR SOME AH-ALGEBRAS
}

\author{
CORNEL PASNICU ${ }^{1}$
}

In this note we prove that "many" unital AH-algebras (which may not be of real rank zero) have cancellation,(SC) and that their $K_{0}$-group is weakly unperforated in the sense of G. A. Elliott. A new characterization of the AH-Algebras of real rank zero and with the dimensions of their local spectra uniformly bounded $(\leqq 3)$ is given.

\section{Introduction.}

In this note we consider $C^{*}$-algebras that can be represented as inductive limits $A=\lim \left(A_{n}, \Phi_{n, m}\right)$ of what we shall call trivial locally homogeneous $C^{*}$-algebras $A_{n}=\vec{\oplus}_{i=1}^{s_{n}} P_{n, i} M_{[n, i]}\left(C\left(X_{n, i}\right)\right) P_{n, i}$, where $X_{n, i}$ are finite connected CW complexes and $P_{n, i}$ are projections. Following a terminology introduced in [4] we shall call these $C^{*}$-algebras $\mathrm{AH}$ algebras (approximately homogeneous). E. G. Effros raised in [8] the problem of finding suitable invariants for $\mathrm{AH}$ algebras. We prove that "many" unital AH algebras $A$ have cancellation and also have (SC)(strict comparability), i.e. for any two projections $p$ and $q$ in $A$ such that $\tau(p)<\tau(q)$ for any tracial state $\tau$ of $A$ it follows that $p$ is (Murray-von Neumann) equivalent to a proper subprojection of $q$ (see Theorem 3.1). If $A$ is a addition simple, then $K_{0}(A)$ is weakly unperforated (see Corollary 3.2). In fact, as we point out, $K_{0}(A)$ is weakly unperforated, but in the sense of Elliott [9], even when $A$ is not simple. These results give new particular affirmative answers to a conjecture of B. Blackadar in [3]. For related results see [2], [4], [1], [20], [21], [7], [18], [14], [15] and [16]. The proofs of our above results rely on the new idea of using the fact that (SC) and cancellation are shape invariant (see Lemma 2.5) and they combine [11, Lemma 2.3, Remark 2.4 and Lemma 2.13] with techniques from [18].

The class of unital $\mathrm{AH}$ algebras which are proved in Theorem 3.1 to have cancellation and (SC) contains e.g. all the unital, real rank zero $\mathrm{AH}$ algebras $A=\lim _{\rightarrow} A_{n}^{\prime}$, where the spectra of the trivial locally homogeneous algebras $A_{n}^{\prime}$ are uniformly bounded. This follows immediately from [22] and [11, Remark 1.4.6]. In our Theorem 3.3 we point out a new characterization of the real rank zero

\footnotetext{
1 This research was supported by NSF grant DMS-9401515.

Received August 29, 1994.
} 
inductive limits of trivial locally homogeneous $C^{*}$-algebras whose spectra have uniformly bounded dimensions (ฏ3) (see also [6] and [13]) which have been intensively studied by Elliott and Gong in their remarkable classification paper [11]. This characterization is an asymptotic condition in terms of the spectrum variation of the connecting homomorphisms and of the dimensions of the spectra of the building blocks in an appropriate inductive system of the $C^{*}$-algebra (in fact, it is a similar condition with the hypothesis of Theorem 3.1, but stronger in the unital case).

The results proved in this note can be used, in particular, to identify $C^{*}$-algebras which do not belong to "large" classes of $\mathrm{AH}$ algebras and also they are related to Elliott's project of the classification of the separable, nuclear $C^{*}$-algebras ([10]).

\section{Preliminaries.}

Let $A$ be a unital $C^{*}$-algebra. We shall denote by $T(A)$ the set of all tracial states on $A$. We shall say that $p \in A$ is a projection if $p=p^{*}=p^{2}$. For two projections $p$, $q \in A$ we shall write $p \prec q$ if $p$ is equivalent to a proper subprojection of $q$, i.e. ( $\exists) \in A$ such that $v^{*} v=p$ and $v v^{*}<q$. We shall say that $K_{0}(A)$ is weakly unperforated if whenever $n \cdot x>0$ for some $x \in K_{0}(A)$ and some positive integer $n$, it follows that $x>0$. For the definition and some properties of the spectrum variation $\operatorname{SPV}(\Phi)$ of a homomorphism $\Phi$ between two trivial homogeneous $C^{*}$-algebras (with metrizable spectra) see [11].

In what follows we shall need the following results and definitions:

THEOREM 2.1 (see [11, Lemma 2.3 and Remark 2.4]). Let $X$ be a connected finite $\mathrm{CW}$ complex with metric $d, F \subset C(X)$ be a finite set. For any integer $N$ and $\varepsilon>0$, there is $\delta>0$ such that, if $\Phi: C(X) \rightarrow P M_{k}(C(Y)) P$ is a unital homomorphism with $\operatorname{SPV}(\Phi)<\delta$, where $Y$ is a connected finite $\mathrm{CW}$ complex and $P \in M_{k}(C(Y))$ is a projection, then one of the following two statements is true:

(a) $\operatorname{rank}(P) \geqq N$

(b) There are $p_{1}, p_{2}, \ldots, p_{n} \in P M_{k}(C(Y)) P$ with $\sum_{i} p_{i}=P$ and

$$
\left\|\Phi(f)-\sum_{i=1}^{n} f\left(x_{i}\right) p_{i}\right\|<\varepsilon \text { for all } f \in F
$$

and furthermore, $\Phi$ is homotopic to $\Phi^{\prime}$ defined by

$$
\Phi^{\prime}(f)=\sum_{i=1}^{n} f\left(x_{i}\right) p_{i}
$$

The following is also true: $\operatorname{SPV}(\Phi)<\frac{\delta}{\ell}$ implies either $\left(\mathrm{a}^{\prime}\right) \operatorname{rank}(P) \geqq \ell N$ or $(\mathrm{b})$ as above, 
Theorem 2.2 (see [22, Theorem 2.5]). Let $A$ be a unital inductive limit of $\left(A_{n}, \Phi_{n, m}\right)$ with $A_{n}=\bigoplus_{i=1}^{k_{n}} M_{[n, i]}\left(C\left(X_{n, i}\right)\right)$, where $X_{n, i}$ are path connected metrizable spaces and $[n, i]$ are integers. If $A$ is of real rank zero, then for any given $n$ and $\varepsilon>0$, there is an $m>n$ such that any partial homomorphism

$$
\Phi_{n, m}^{i, j}: M_{(n, i]}\left(C\left(X_{n, i}\right)\right) \rightarrow M_{[m, j]}\left(C\left(X_{m, j}\right)\right)
$$

of $\Phi_{n, m}$ satisfies:

$$
\operatorname{SPV}\left(\Phi_{n, m}^{i, j}\right)<\varepsilon .
$$

The following lemma is a result of K. R. Goodearl ([16]) and N. C. Phillips ([19]):

LEMMA 2.3. Let $X$ be a compact space of dimension d and let $p, q$ be projections in $C\left(X, M_{n}\right)$. If $\operatorname{rank}(q(x))-\operatorname{rank}(p(x)) \geqq \max \{(d-1) / 2,1\}$ for all $x \in X$, then $p \prec q$.

The following simple result is a slight improvement of Lemma 3.2 in [18] and its proof, which will be not given, follows an idea in [2]:

Lemma 2.4. Let $A=\lim _{\rightarrow}\left(A_{n}, \Phi_{n, m}\right)$, where the $C^{*}$-algebras $A_{n}$ are unital and have tracial states and the connecting homomorphisms $\Phi_{n, m}: A_{n} \rightarrow A_{m}$ are unital.

Let $p$ and $q$ be projections in some $A_{n}$ such that $\sigma(\tilde{p})<\sigma(\tilde{q})$ for any $\sigma \in T(A)$, where $\tilde{p}(\operatorname{resp} . \tilde{q})$ is the canonical image of $p(\operatorname{resp} . q)$ in the inductive limit $A=\lim _{\rightarrow}\left(A_{n}, \Phi_{n, m}\right)$. Then there is $m>n$ such that:

$$
\tau\left(\Phi_{n, m}(p)\right)<\tau\left(\Phi_{n, m}(q)\right)
$$

for any $\tau \in T\left(A_{m}\right)$.

Definition (compare with [3]). A unital $C^{*}$-algebra $A$ is said to have (SC) (strict comparability) if $T(A) \neq \phi$ and whenever $p$ and $q$ are projections in $A$ with $\tau(p)<\tau(q)$ for any $\tau \in T(A)$ it follows that $p \prec q$.

The next proposition is a slight extension of a result in [18] and has a similar proof (which will be not given):

Proposition 2.5 (see [18, Proposition 3.13 and its proof]). Let $A=\lim _{\rightarrow}\left(A_{n}, \Phi_{n}\right)$ and $B=\lim _{\rightarrow}\left(B_{n}, \Psi_{n}\right)$, where $A_{n}, B_{n}$ are arbitrary unital $C^{*}$-algebras and the connecting homomorphisms $\Phi_{n}, \Psi_{n}$ are unital.

Suppose that there is an EP-commutative diagram (see $[18,2.3])$ with unital homomorphisms $\alpha_{n}$ and $\beta_{n}(n \geqq 1)$ :

$$
\begin{gathered}
A_{1} \stackrel{\Phi_{1}}{\longrightarrow} A_{2} \stackrel{\Phi_{2}}{\longrightarrow} A_{3} \stackrel{\Phi_{3}}{\longrightarrow} A_{4} \stackrel{\Phi_{4}}{\longrightarrow} \cdots \\
\alpha_{1} \backslash \beta_{1} \nearrow \alpha_{2} \searrow \beta_{2} \nearrow \alpha_{3} \backslash \beta_{3} \nearrow \\
B_{1} \stackrel{\Psi_{1}}{\longrightarrow} B_{2} \stackrel{\Psi_{2}}{\longrightarrow} B_{3} \stackrel{\Psi 3}{\longrightarrow} \cdots
\end{gathered}
$$


(note that this happens if e.g. the above diagram is commutative at the level of homotopy). Then:

a) $T(A) \neq \phi \Leftrightarrow T(B) \neq \phi$

b) $A$ has (SC) $\Leftrightarrow B$ has (SC).

If furthermore the above diagram is a stably EP-commutative diagram (that is, after taking the tensor product with $M_{n}$ for any $n$, it is still an EP-commutative diagram) then:

c) $A$ has cancellation $\Leftrightarrow B$ has cancellation.

Definition (compare with [7,2.1.8]). Let $A=P M_{k}(C(X)) P$ and $B=$ $Q M_{\ell}(C(Y)) Q$ be homogeneous algebras, where $X$ and $Y$ are compact and connected and $P$ and $Q$ are projections. A *-homomorphism $\Phi: A \rightarrow B$ is said to be large if

a) $\Phi=0$

or

b) $\Phi(P)=Q$ and $\operatorname{rank}(\Phi(P)) \geqq \max \{\operatorname{dim}(Y) / 2,1\} \operatorname{rank}(P)$.

\section{The main results.}

THEOREM 3.1. Let

$$
A=\lim _{\rightarrow}\left(A_{n}, \Phi_{n, m}\right)
$$

where $A_{n}=\oplus_{i=1}^{s_{n}} P_{n, i} M_{[n, i]}\left(C\left(X_{n, i}\right)\right) P_{n, i}, X_{n, i}$ are finite connected $\mathrm{CW}$ complexes, $P_{n, i} \in M_{[n, i]}\left(C\left(X_{n, i}\right)\right)$ are projections and $\Phi_{n, m}$ are unital. Let $\delta(n, i)$ be the largest strictly positive number (perhaps $+\infty)$ (depending on $X_{n, i}$ only) $\delta$ such that $B(x ; a)$ is contractible for any $a<2 \delta$ and any $x \in X_{n, i}$, where $B(x ; a)$ denotes the closed ball in $X_{n, i}$ of center $x$ and radius $a$. Suppose that for any given positive integer $n$ there is $m>n$ such that any partial homomorphism

$$
\Phi_{n, m}^{i . j}: P_{n, i} M_{[n, i]}\left(C\left(X_{n, i}\right)\right) P_{n, i} \rightarrow P_{m, j} M_{[m, j]}\left(C\left(X_{m, j}\right)\right) P_{m, j}
$$

of $\Phi_{n, m}$ satisfies:

$$
\operatorname{SPV}\left(\Phi_{n, m}^{i, j}\right)<\frac{\delta\left(n_{1}, i\right)}{\max \left\{\operatorname{dim}\left(X_{m, j}\right) / 2,1\right\}}
$$

Then:

a) $A$ has (SP) (see Preliminaries for definition).

b) A has cancellation.

Proof. a) From the proof of [11, Lemma 2.3] it follows that (for any $n$ and $i$ ) either $\delta(n, i)$ is a positive number $\delta$ as in [11, Lemma 2.3], corresponding to $X=X_{n, i}, F=\phi$, and $N=\varepsilon=1$ or $\delta(n, i)=+\infty$. Let us introduce for any $n$ and $i$ the usual notation $A_{n}^{i}=P_{n, i} M_{[n, i]}\left(C\left(X_{n, i}\right)\right) P_{n, i}$. Using now the hypothesis, it 
follows that there is $n_{2}>n_{1}=1$ such that all the partial homomorphisms $\Phi_{1, n_{2}}^{i, j}: A_{1}^{i} \rightarrow A_{n_{2}}^{j}$ satisfy:

$$
\operatorname{SPV}\left(\Phi_{1, n_{2}}^{i, j}\right)<\frac{\delta(1, i)}{\max \left\{\operatorname{dim}\left(X_{n_{2}, j}\right) / 2,1\right\}}
$$

By Theorem 2.1 it follows that for any $i, j$ either

$$
\Phi_{1, n_{2}}^{i, j}: A_{1}^{i} \rightarrow \Phi_{1, n_{2}}^{i, j}\left(1_{A_{1}^{i}}^{i}\right) A_{n_{2}}^{j} \Phi_{1, n_{2}}^{i, j}\left(1_{A_{1}^{i}}\right)
$$

is large (see Preliminaries for definition) or $\Phi_{1, n_{2}}^{i, j}: A_{1}^{i} \rightarrow \Phi_{1, n_{2}}^{i, j}\left(1_{A_{1}^{i}}\right) A_{n_{2}}^{j} \Phi_{1, n_{2}}^{i, j}\left(1_{A_{1}^{i}}\right)$ is homotopic to a unital *-homomorphism $A_{1}^{i} \rightarrow \Phi_{1, n_{2}}^{i, j}\left(1_{A_{1}}{ }^{i}\right) A_{n_{2}}^{j} \Phi_{1, n_{2}}^{i, j}\left(1_{A_{1}^{i}}\right)$ with finite dimensional image. Hence there is a diagram:

$$
\begin{gathered}
A_{1} \stackrel{\Phi_{1, n_{2}}}{\longrightarrow} A_{n_{2}} \\
\alpha_{1} \searrow \nearrow_{\beta_{1}} \\
B_{1}
\end{gathered}
$$

where:

i) $\alpha_{1}$ and $\beta_{1}$ are unital *-homomorphisms

ii) $B_{1}=\bigoplus_{j=1}^{t_{1}} B_{1}^{j}$ where for any $j B_{1}^{j}=Q_{j} M_{m_{j}}\left(C\left(Z_{j}\right)\right) Q_{j}$ with $Z_{j}$ a finite connected CW complex and $Q_{j}$ a projection in $M_{m_{j}}\left(C\left(Z_{j}\right)\right)$

iii) any partial homomorphism $A_{1}^{i} \rightarrow\left(\alpha_{1}\right)^{i, j}\left(1_{A_{1}}\right) B_{1}^{j}\left(\alpha_{1}\right)^{i, j}\left(1_{A_{1}}\right)^{i}$ induced by $\alpha_{1}$ is either large of surjective with finite dimensional image.

iv) the above diagram (1) is commutative within homotopy.

Continuing in this way, we construct inductively a diagram of unital *-homomorphisms:

$$
\begin{aligned}
& A_{1} \stackrel{\Phi_{n_{1}, n_{2}}}{\longrightarrow} A_{n_{2}} \stackrel{\Phi_{n_{2}, n_{3}}}{\longrightarrow} A_{n_{3}} \rightarrow \cdots \rightarrow A_{n_{m}} \stackrel{\Phi_{n_{m}, n_{m+1}}}{\longrightarrow} A_{n_{m+1}} \rightarrow \cdots \\
& \alpha_{1} \searrow / \beta_{1} \alpha_{2} \searrow / \beta_{2} \quad \alpha_{m} \searrow \quad \beta_{m} \\
& B_{1} \quad B_{2} \quad B_{m}
\end{aligned}
$$

which commutes within homotopy and each diagram:

$$
\begin{gathered}
A_{n_{m}} \stackrel{\Phi_{n_{m}, n_{m+1}}}{\longrightarrow} A_{n_{m+1}} \\
\alpha_{1} \searrow \underset{\beta_{1}}{{ }_{B_{m}}}
\end{gathered}
$$

has the above properties i)-iv). Using Proposition 2.5 and supposing that $n_{m}=m, m \geqq 1$ (to save the notation), we may assume that $A$ is the $C^{*}$-inductive limit of the system:

$$
A_{1} \stackrel{\alpha_{1}}{\longrightarrow} B_{1} \stackrel{\beta_{1}}{\longrightarrow} A_{2} \stackrel{\alpha_{2}}{\longrightarrow} B_{2} \stackrel{\beta_{2}}{\longrightarrow} A_{3} \longrightarrow \ldots \longrightarrow A_{n} \stackrel{\alpha_{n}}{\longrightarrow} B_{n} \stackrel{\beta_{n}}{\longrightarrow} A_{n+1} \longrightarrow \ldots
$$

Now let $P$ and $Q$ be projections in $A$ with $\tau(P)<\tau(Q)$ for any $\tau \in T(A)$. By 
simple approximation arguments and changing the representatives in the inductive limit, since $A=\overline{\bigcup_{n=1}^{\infty} \mu_{n, \infty}\left(A_{n}\right)}$, we may suppose that $P=\mu_{1, \infty}(p)$, $Q=\mu_{1, \infty}(q)$ for some projections $p$ and $q$ in $A_{1}$. Here $\mu_{n, \infty}: A_{n} \rightarrow A=$ $\lim _{\rightarrow}\left(A_{m}, \beta_{m} \circ \alpha_{m}\right)$ are the canonical *-homomorphisms.

Considering $A=\lim _{\rightarrow}\left(A_{n}, \beta_{n} \circ \alpha_{n}\right)$ and applying Lemma 2.4, we may suppose that:

$$
\sigma(p)<\sigma(q) \text { for any } \sigma \in T\left(A_{1}\right)
$$

Let $p=\oplus_{k=1}^{s_{1}} p_{k}, q=\oplus_{k=1}^{s_{1}} q_{k} \in A_{1}=\bigoplus_{k=1}^{s_{1}} A_{1}^{k}$ where $A_{1}^{k}=$ $P_{1, k} M_{[1, k]}\left(C\left(X_{1, k}\right)\right) P_{1, k}$. By (2) it follows that:

$$
\operatorname{rank}\left(p_{k}\right)<\operatorname{rank}\left(q_{k}\right), \quad 1 \leqq k \leqq s_{1}
$$

Let $\left(\alpha_{1}\right)^{i, j}: A_{1}^{i} \rightarrow\left(\alpha_{1}\right)^{i, j}\left(1_{A_{1}^{i}}\right) B_{1}^{j}\left(\alpha_{1}\right)^{i, j}\left(1_{A_{1}}\right)$ be the partial homomorphism induced by $\alpha_{1}$. It can be shown (using [11, Lemma 2.13] and [18]) that there is an integer $k_{i, j} \geqq 0$ such that for any projection $r$ in $A_{1}^{i}$ we have:

$$
\operatorname{rank}\left(\left(\alpha_{1}\right)^{i, j}(r)\right)=k_{i, j} \cdot \operatorname{rank}(r)
$$

We have to consider three cases for $i, j$ fixed:

I) If $k_{i, j}=0$, then $\left(\alpha_{1}\right)^{i, j}\left(p_{i}\right)=\left(\alpha_{1}\right)^{i, j}\left(q_{i}\right)=0$

II) If $k_{i, j}>0$ and $\left.\left.\left(\alpha_{1}\right)^{i, j}: A_{1}^{i} \rightarrow\left(\alpha_{1}\right)^{i, j}\left(1_{A_{1}}\right)\right) B_{1}^{j}\left(\alpha_{1}\right)^{i, j}\left(1_{A_{1}}\right)^{i}\right)$ is surjective with finite dimensional image, then using [11, Lemma 2.13] and [18] it is not difficult to see that $\left(\alpha_{1}\right)^{i, j}\left(p_{i}\right) \prec\left(\alpha_{1}\right)^{i, j}\left(q_{i}\right)$ (we use (3)).

III) If $k_{i, j}>0$ and $\left(\alpha_{1}\right)^{i, j}: A_{1}^{i} \rightarrow\left(\alpha_{1}\right)^{i, j}\left(1_{A_{1}^{i}}\right) B_{1}^{j}\left(\alpha_{1}\right)^{i, j}\left(1_{A_{1}^{i}}\right)$ is large, then, using (4) and (3), we have:

$$
\begin{gathered}
\operatorname{rank}\left(\left(\alpha_{1}\right)^{i, j}\left(q_{i}\right)\right)-\operatorname{rank}\left(\left(\alpha_{1}\right)^{i, j}\left(p_{i}\right)\right)=k_{i, j}\left(\operatorname{rank}\left(q_{i}\right)-\operatorname{rank}\left(p_{i}\right)\right) \\
\geqq k_{i, j} \geqq \max \left\{\operatorname{dim}\left(Z_{j}\right) / 2,1\right\}
\end{gathered}
$$

By Lemma 2.3 this implies that:

$$
\left(\alpha_{1}\right)^{i, j}\left(p_{i}\right) \prec\left(\alpha_{1}\right)^{i, j}\left(q_{i}\right) \text { in } B_{1}^{j}
$$

In conclusion (using also the fact that $A$ has (SC)), it follows that $P=\mu_{2, \infty}\left(\beta_{1} \circ \alpha_{1}(p)\right)<Q=\mu_{2, \infty}\left(\beta_{1} \circ \alpha_{1}(q)\right)$.

b) Working as in the proof of a) we may suppose that every unital partial homomorphism induced by each $\Phi_{n, n+1}$ is either large (see Preliminaries) or homotopic to a homomorphism with finite dimensional image. Now the result follows using Lemma 2.5c) and stability results for vector bundles in [17].

COROLlary 3.2. Let $A$ be as in Theorem 3.1. If in addition we assume that $A$ is simple, then $K_{0}(A)$ is weakly unperforated. 
Proof. Observe firstly that because $A$ is in addition a simple $C^{*}$-algebra, for any two projections $p$ and $q$ in $A$ we have:

$$
p \prec q \quad \text { in } A \Leftrightarrow \tau(p)<\tau(q) \text { for any } \tau \in T(A) .
$$

Since by Theorem $3.1 A$ has cancellation, in order to prove that $K_{0}(A)$ is weakly unperforated, it is enough to prove that if for some projections $p$ and $q$ in some matrix algebra $B$ over $A$ and some positive integer $n$ we have:

$$
n \cdot p=\underbrace{p \oplus p \oplus \ldots \oplus p}_{n \text { times }} \prec n \cdot q=\underbrace{q \oplus q \oplus \ldots \oplus q}_{n \text { times }}
$$

in $M_{n}(B)$, then:

$$
p \prec q \text { in } B
$$

Let $\tau \in T(B)$. Then $\sigma=\tau \otimes \operatorname{tr}_{n} \in T\left(B \otimes M_{n}\right)$ if $\operatorname{tr}_{n}$ is the (unique) tracial state on $M_{n}$. By the above remark, $(*)$ implies that:

$$
\sigma(n \cdot p)=\tau(p)<\sigma(n \cdot q)=\tau(q)
$$

Since $\tau \in T(B)$ is arbitrary, by Theorem 3.1 a), it follows that $(* *)$ is true.

REMARK. If one replaces the above weakly unperforated property in Corollary 3.2 by the one in the sense of Elliott ([9]), one can obtain the result without the restriction that $A$ be simple. The proof goes using some of the arguments from the proof of Theorem 3.1.

The next result gives a new description of the $\mathrm{AH}$ algebras $A=\lim _{\rightarrow} A_{n}$ with real rank zero for which the dimensions of the spectra of the trivial locally homogeneous algebras $A_{n}$ are $\leqq 3$ (see Theorem 3.3). Note that by a recent remarkable result proved by M. Dadarlat [6] and by Gong [13] it is known that this class of $C^{*}$-algebras coincides with the class of the $\mathrm{AH}$ algebras of real rank zero which are inductive limits of trivial locally homogeneous algebras with the dimensions of their spectra uniformly bounded. Our description is in terms of the spectrum variation of the connecting homomorphisms and of the dimensions of the spectra of the building blocks in an appropriate inductive system of the $C^{*}$-algebra (in fact is a similar condition with that in Theorem 3.1, but stronger in the unital case). In the simple case, by a remarkable result of Elliott and Gong in [11], this class of $C^{*}$-algebras is classified by the graded, scaled, ordered $K_{*}$ group.

THEOREM 3.3. Let $A$ be a $C^{*}$-algebra. The following are equivalent:

a) $A$ can be written

$$
A=\lim _{\rightarrow}\left(A_{n}, \Phi_{n, m}\right),
$$

where $A_{n}=\bigoplus_{i=1}^{s_{n}} P_{n, i} M_{[n, i]}\left(C\left(X_{n, i}\right)\right) P_{n, i}, X_{n, i}$ are finite connected $\mathrm{CW}$ complexes, 
$[n, i], s_{n}$ are positive integers and $P_{n, i} \in M_{[n, i]}\left(C\left(X_{n, i}\right)\right)$ are projections such that:

For any given $\varepsilon>0$ and any given positive integer $n$, there is $m_{0}>n$ such that any partial homomorphism

$$
\Phi_{n, m}^{i, j}: P_{n, i} M_{[n, i]}\left(C\left(X_{n, i}\right)\right) P_{n, i} \rightarrow P_{m, j} M_{[m, j]}\left(C\left(X_{m, j}\right)\right) P_{m, j}
$$

of $\Phi_{n, m}: A_{n} \rightarrow A_{m}$ with $m \geqq m_{0}$ satisfies

$$
\operatorname{SPV}\left(\Phi_{n, m}^{i, j}\right)<\frac{\varepsilon}{\operatorname{dim}\left(X_{m, j}\right)+1}
$$

b) $A$ has real rank zero and $A=\lim _{\rightarrow}\left(A_{n}^{\prime}, \Psi_{n, m}\right)$ where

$$
A_{n}^{\prime}=\oplus_{i=1}^{t_{n}} Q_{n, i} M_{\{n, i\}}\left(C\left(Y_{n, i}\right)\right) Q_{n, i}
$$

$Y_{n, i}$ are connected $\mathrm{CW}$ complexes of dimension $\leqq 3, H^{3}\left(Y_{n, i}\right)$ are finite, $\{n, i\}, t_{n}$ are positive integers and $Q_{n, i} \in M_{\{n, i\}}\left(C\left(Y_{n, i}\right)\right)$ are projections.

Proof. a) $\Rightarrow$ b). As in the proof of [11, Corollary 2.25] it follows that $A$ has real rank zero. Now, the proof goes as in the proof of Theorem 3.2 in [6] (the case when $\left.\sup _{n, i}\left(\operatorname{dim}\left(X_{n, i}\right)\right)<+\infty\right)$ using the hypothesis and Theorem 2.1 (i.e. [11, Lemma 2.3 and Remark 2.4]) instead of the fact that the real rank of $A$ is zero, of Theorem 2.2 (i.e. [22, Theorem 2.5]) and [11, Remark 1.4.6] and of [11, Lemma 2.3] (to get $m$-large *-homomorphisms in the sense of [7] or *-homomorphisms with finite dimensional irnage) and observing that the appropriate variants of [11, Theorem 2.29 and Remark 2.30] hold if we replace the conditions that $A$ has real rank zero and $\sup _{n, i}\left(\operatorname{dim}\left(X_{n, i}\right)\right)<+\infty$ by our hypothesis.

b) $\Rightarrow$ a). Let $\varepsilon>0$ and let a positive integer $n$ be fixed. Then, by Theorem 2.2 and [11, Remark 1.4.6], there is $m_{0}>n$ such that for any partial homomorphism $\Psi_{n, m}^{i, j}$ we have:

$$
\operatorname{SPV}\left(\Psi_{n, m}^{i, j}\right)<\frac{\varepsilon}{4} \quad \text { for any } \quad m \geqq m_{0} .
$$

On the other hand, the hypothesis obviously implies that:

$$
\frac{\varepsilon}{4} \leqq \frac{\varepsilon}{\operatorname{dim}\left(Y_{m, j}\right)+1} .
$$

Hence:

$$
\operatorname{SPV}\left(\Psi_{n, m}^{i, j}\right)<\frac{\varepsilon}{\operatorname{dim}\left(Y_{m, j}\right)+1}
$$

for all the partial homomorphisms $\Psi_{n, m}^{i, j}$ with $m \geqq m_{0}$.

REMARK. We would like to point out that until now most of the known results about inductive limit $C^{*}$-algebras (concerning real rank, stable rank, exponential rank, cancellation, (SC), classification etc.) have been obtained for $C^{*}$-algebras 
defined by inductive systems with slow dimension growth in one sense or another (exceptions are above, in [12], [18] and eventually in other few places). Note also that the system satisfying the condition a) in the above theorem generally has NO slow dimension growth in any sense, in contrast with the system in Theorem 3.3 b) which defines the same algebra.

\section{REFERENCES}

1. J. Anderson, B. Blackadar and U. Haagerup, Minimal projections in the reduced $C^{*}$-algebra of $Z_{n} * Z_{m}$, J. Operator Theory (to appear).

2. B. Blackadar, Traces on simple AF $C^{*}$-algebras, J. Funct. Anal. 38 (1980), 156-168.

3. B. Blackadar, Comparison theory for simple $C^{*}$-algebras, Operator Algebras and Applications (ed. D. E. Evans and M. Takesaki), L. M. S. Lecture Notes Series 135 (1989), 21-54.

4. B. Blackadar, Matricial and ultramatricial topology, Preprint.

5. L. G. Grown and G. K. Pedersen, $C^{*}$-algebras of real rank zero, J. Funct. Anal. 99 (1991) 131-149.

6. M. Dadarlat, Reduction to dimension three of local spectra of real rank zero $C^{*}$-algebras, Preprint.

7. M. Dadarlat and A. Nemethi, Shape theory and connective K-theory, J. Operator Theory 23 (1990), 207-291.

8. E. G. Effros, Dimensions and C*-algebras, CBMS Regional Conf. Series in Math., vol. 46, Amer. Math. Soc. Providence R.I., 1981.

9. G. A. Elliott, Dimension groups with torsion, Internat. J. Math. Sci. 1 (1990), 361-380.

10. G. A. Elliott, Are amenable $C^{*}$-algebras classifiable? (to appear).

11. G. A. Elliott and G. Gong, On the classification of $C^{*}$-algebras of real rank zero II, Preprint.

12. G. A. Elliott, G. Gong, H. Lin and C. Pasnicu, Abelian $C^{*}$-subalgebras of $C^{*}$-algebras of real rank zero and inductive limit $C^{*}$-algebras, Preprint.

13. G. Gong, On inductive limits of matrix algebras over higher dimensional spaces, Part. II, Preprint.

14. G. Gong and H. Lin, Exponential rank of inductive limit $C^{*}$-algebras, Math. Scand. 71 (1992), 301-319.

15. K. R. Goodearl, Notes on a class of simple $C^{*}$-algebras with real rank zero, Publicacions Mathematiques 36 (1992), 637-654.

16. K. R. Goodearl, Riesz decomposition in inductive limit $C^{*}$-algebras (to appear).

17. D. Husemoller, Fibre Bundles, Springer Verlag, 1966.

18. M. Martin and C, Pasnicu, Some comparability results in inductive limit $C^{*}$-algebras, J. Operator Theory (to appear).

19. N. C. Phillips, The $C^{*}$-projective length of $n$-homogeneous $C^{*}$-algebras, J. Operator Theory (to appear).

20. M. Rieffel, Dimension and stable rank in the $K$-theory of $C^{*}$-algebras, Proc. London Math. Soc. (3) 46 (1983), 301-333.

21. M. Rieffel, The cancellation theorem for projective modules over irrational rotation algebras, Proc. London Math. Soc. (3) 47 (1983), 285-302.

22. H. Su, On the classification of real rank zero $C^{*}$-algebras: inductive limits of matrix algebras over non-Hausdorff graphs, thesis, University of Toronto. 